\title{
Noisy Iris Verification: A Modified Version of Local Intensity Variation Method
}

\author{
Nima Tajbakhsh ${ }^{1}$, Babak Nadjar Araabi ${ }^{1,2}$, and Hamid Soltanian-zadeh ${ }^{1,2,3}$ \\ ${ }^{1}$ Control and Intelligent Processing Center of Excellence, School of Electrical \\ and Computer Engineering, University of Tehran, Iran \\ ${ }^{2}$ School of Cognitive Sciences, IPM, Tehran, Iran \\ ${ }^{3}$ Radiology Image Analysis Lab., Henry Ford Health System, Detroit, MI, USA \\ n.tajbakhsh@ece.ut.ac.ir, \{a.araabi, hszadeh\}@ut.ac.ir
}

\begin{abstract}
In this paper, a modified version of local intensity variation method is proposed to enhance the efficiency of identification system while dealing with degradation factors presented in iris texture. Our contributions to improve the robustness and performance of local intensity variation method consist of defining overlapped patches to compensate for deformation of texture, performing a de-noising strategy to remove high frequency components of intensity signals, proposing to add a coding strategy, and combining the dissimilarity values obtained from intensity signals. Experimental results on UBIRIS database demonstrate the effectiveness of proposed method when facing low quality images. To assess the robustness of proposed method to noise, lack of focus, and motion blur, we simulate these degradation factors that may occur during image acquisition in non-ideal conditions. Our results on a private database show that verification performance remains acceptable while the original method [11] suffers from a dramatic degradation.
\end{abstract}

Keywords: Noisy iris recognition, Score fusion, Robustness evaluation.

\section{Introduction}

Iris as the most promising biometric has attracted attention in recent years. Stability of iris texture along lifetime and its stochastic structure suggest the iris recognition system as a reliable authentication technology. Although several commercial iris recognition systems have been developed, they are to be used under constraints associated with lighting condition and subjects' behavior. In many cases, subjects have to participate in a verification process several times until the image acquisition process manages to capture a high quality iris image. This mainly comes from the fact that the efficiency of the current commercial iris recognition systems is highly affected by the quality of captured images and consequently the detailed information of iris texture. Therefore, this is typical of such systems to ask the subjects to participate in image acquisition process several times. Regardless of hardships that subjects face, sensitivity of the system performance to the quality of the acquired images restricts application of iris recognition in situations where we do not want to make the subjects aware of acquisition of their biometric data or where the aim is to make use of the system in outdoor conditions. 
Recent research efforts in iris recognition have aimed to relax mentioned constraints. Many recent works with aim of addressing the challenges caused by low quality images have been evaluated on the UBIRIS database [1]. In the following, we briefly point to them and discuss about their results and achievements.

In [2], color Information instead of texture information is proposed to make more reliable decisions dealing with the low quality images. Although the color information on the first session of the UBIRIS outperforms the methods that are merely based on texture information, its performance declines significantly when facing the images of the second session of the UBIRIS. The main reason for lack of performance of colorbased information may originate from the fact in uncontrolled lighting conditions, the color content of iris texture influenced and altered by the source of environmental illumination.

In [3], the contour of the shapes of the iris stroma has been exploited to obtain a signature robust to noise factors. Their method especially in small training samples does not provide high efficiency. In [4], an iris coding robust to noisy environment has been proposed in which the entropy of consecutive overlapped angular patches of normalized iris is computed to form a one-dimensional signature for each captured iris image. In spite of effectiveness of this approach, comparison of two signatures in this algorithm is a time-consuming process. In [5], to give a robust representation of iris texture, a division strategy similar to that proposed in [6] has been adopted to reduce the bad effects of eyelids and eyelashes to a minimum in feature extraction stage. Then, an independent feature extraction strategy is performed on each subregion in which the Local Edge Pattern (LEP) operator is applied to extract various edge or corner patterns. In spite of promising performance, this method has not been evaluated on the second session of the UBIRIS database which is comprised of a large number of blurred or highly occluded images.

In [7], a novel prototype iris recognition system (Eagle-EyesTM) for long-range human identification is developed. Eagle-EyesTM is a multi-biometric system that acquires, a face and two iris images from subjects presenting anywhere within its large capture volume. In our previous works [8,9], texture information of visible and near infrared iris images are combined to enhance verification and recognition results obtained from poor quality images.

In this work, our aim is to extract local intensity variation in a way that its information content less affected by noisy regions. In this way, authentication system will be capable of facing highly occluded images even those captured in uncontrolled lighting conditions. The main ideas are proposing a de-noising strategy prior to feature extraction and a decentralized decision-making process in matching phase.

The rest of this paper is organized as follows: Section 2 describes the local intensity variation followed by a brief analysis on its disadvantages. A detailed description of the proposed feature extraction method is given in Section 3. Section 4 reports the experimental results and, finally, Section 5 presents the conclusions.

\section{Local Intensity Variation Method}

The idea of analyzing local intensity variations along angular direction is introduced in $[10,11]$. Since the most discriminative information of iris texture tends to run in the 
radial direction, examining variations in the angular direction provides desirable features of iris texture [11]. Ma et al. [10,11] propose two well-known feature extraction techniques based on the local intensity variation. In both of them, the main idea is to extract sharp variations of intensity signals although different approaches are employed. However, the sharp variations of intensity signals are highly affected by noise factors presented in the iris texture. In [10], key local sharp variation points are extracted to represent the appearance or disappearance of important image structures. Determining the positions of key local points is carried out through finding local extreme, minimum or maximum in detail components of decomposed 1-D intensity signals. However, based on the quality of captured images it is possible that local extreme points vanish or some other spurious ones created along intensity signals. In [11], to capture details of the iris texture, Gaussian-Hermite moments of intensity signals are calculated. Due to high frequency components of intensity signals, the Gaussian-Hermite moment kernels are adopted to reflect random shape information of the iris. However, efficiency of the shape information extracted from the intensity signals is greatly influenced by those regions occluded by eyelids and eyelashes. Regardless of this issue, sharp variations of intensity signals playing a crucial role in the feature extraction strategy, perhaps, cannot be obtained from the images captured with poor focus.

\section{Proposed Method}

In this work, we make four contributions to local intensity variation including defining overlapped patches to compensate for deformation of texture, performing a de-noising strategy for removing high frequency component of intensity signals, proposing to add a coding strategy, and combining the dissimilarity values obtained from intensity signals to make more reliable decisions. This section begins by describing preprocessing stage and then the feature extraction is introduced.

\subsection{Preprocessing}

In [12], it is shown that isolating the eyelids and eyelashes and replacing the missing iris information through interpolation of the noise-free regions will not result in a significant increment in the separability between the intra- and inter-class comparisons. On the other hand, eyelids and eyelashes can transform the shape of intensity signals in a way that even removing the high frequency components -as a de-noising strategy- will not restore the main information of signals in those regions. Therefore, to eliminate such adverse effects, the upper half of iris that is usually obstructed by noisy regions is discarded [13]. As a result, all of the normalized images are of resolution 256x64 pixels.

\subsection{Feature Extraction}

The main idea is to extract a number of intensity signals that are capable of facing deformed texture and noisy regions. The intensity signals are constructed by averaging the gray levels of overlapped patches along radial direction. Selecting overlapped patches makes it possible to handle deformed texture. Here, instead of 
sharp variations, our goal is to extract the soft variations or in other words, the main behavior of intensity signals. In this way, the captured information is less affected by noisy regions of the texture. Accordingly, we apply the wavelet de-noising to eliminate sharp variations of intensity signals. Moreover, this process enables the information content to be concentrated at a specific level of wavelet decomposition. Using Discrete Wavelet Transform (DWT), one can obtain scale of wavelet function with most informative coefficients. Since the main information of de-noised signals lies in low frequency components; and regarding to the fact that DWT cannot provide enough discriminative coefficients at large scales, the Continues Wavelet Transform (CWT) is adopted to extract features from de-noised signals. Thereafter, a coding strategy based on the signs of the coefficients is performed. That is, the positive coefficients are replaced by one and the negative values by zeros. Therefore, a binary code corresponding to every intensity signal is generated. In view of the fact that texture information is not uniformly distributed over the texture, the generated binary codes which reflect iris information in different regions have to be incorporated into the decision making process in a weighted manner. Furthermore, such decentralized decision-making minimizes the effects of noise factors (iris obstructions and reflections) through splitting the noisy areas into some sub-regions covered by different intensity signals. The way of combining the dissimilarity values is determined based on the power of discrimination of every intensity signal and their associated probability of corruption by noisy regions. Therefore, this strategy can serve as a complementary scheme to cope with noisy and degraded iris images. Combination of dissimilarities can be performed in several ways. Two common approaches that suggested for combining dissimilarity values are (1) performing a classification task in distance space [14, 15, and 16] and (2) estimating density of dissimilarities to form a fusion rule in probability space $[17,18]$. In this work, we restrict ourselves to the first approach and adopt Support Vector Machines (SVM) as a candidate of linear and nonlinear classifiers to perform classification in distance space. This is due to the fact that mapping observations from distance space into probability space may result in missing information. For example, it is likely to have a meaningful manifold in distance space that enables us to reduce dimension of distance vectors while improving separability of genuine and imposter classes. However, such valuable property may not exist in probability space. An overview of the feature extraction and decision-making processes is depicted in Figure 1.

\section{Experiments}

To enable the effective test of proposed iris recognition method, we evaluate the efficiency of proposed method on the both sessions of UBIRIS database, while the training stage is carried out on a private database (UTIRIS) consists of two sessions with 1540 noisy images, 770 captured in Visible Light (VL) and 770 in Near Infrared (NIR) illumination. There are few images in UBIRIS database which are discarded during the evaluation of proposed method. Some of them failed in image segmentation process and others were not of quality to be involved in verification process. At last, we used 1740 out of 1877 images for performing evaluation of proposed method. 

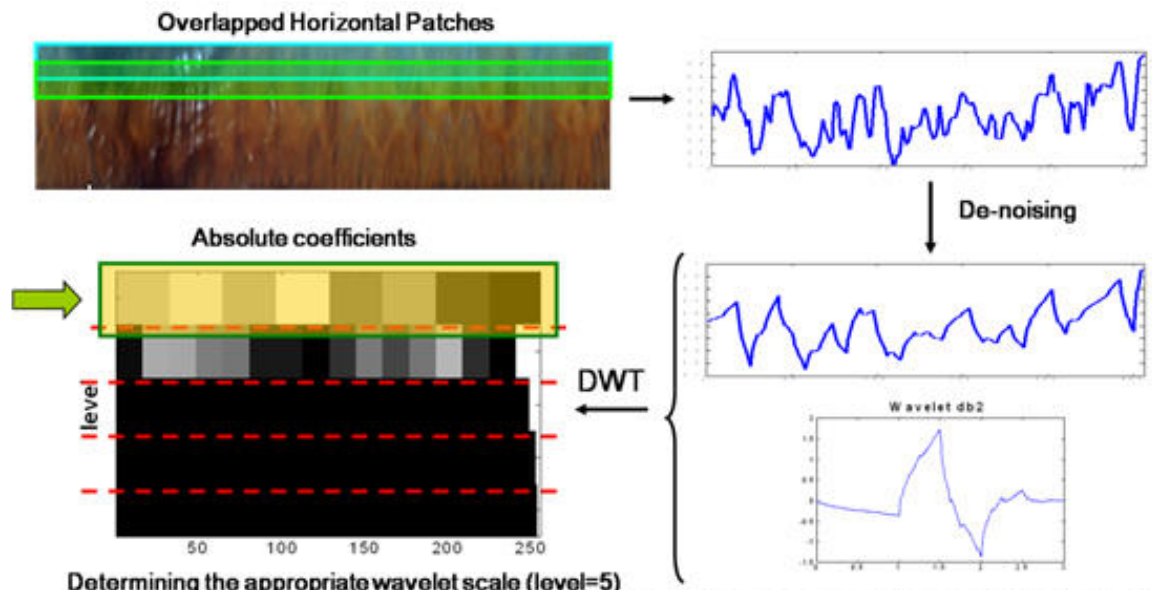

Selecting the appropriate wavelet function (db2)
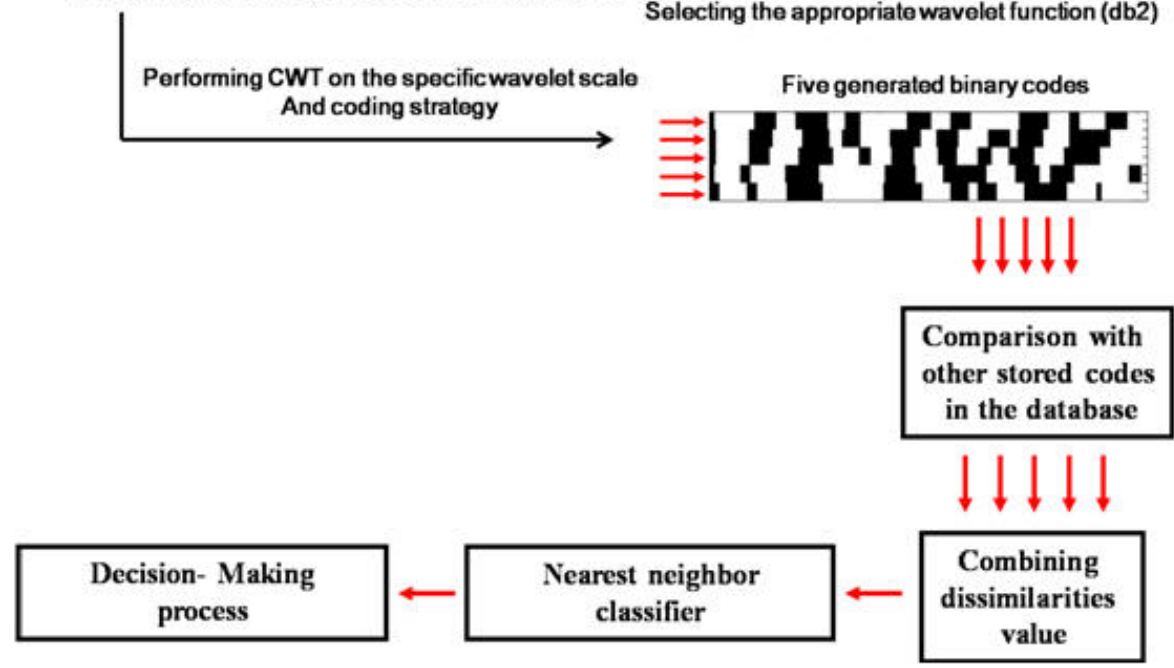

Fig. 1. An overview of proposed method

\subsection{Results}

The training of proposed method is carried out in two stages. The first one is determining the parameters involved in feature extraction which is performed on VL images of the UTIRIS database, and the other is defining the optimal fusion rule which is accomplished by cross validation test on UBIRIS database.

After extensive experiments, horizontal overlapped patches with 15 pixels width and 10 overlapped pixels were chosen. This enabled extraction of five intensity signals from a given iris pattern. The de-noising strategy was accomplished by performing wavelet decomposition at level 3 and reconstructing the signal just based 


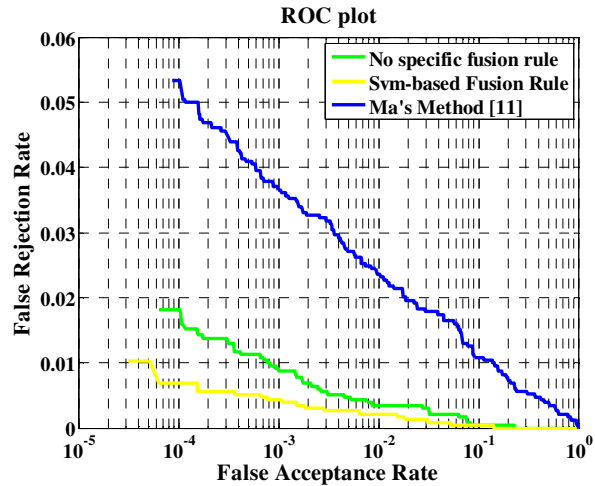

(a)

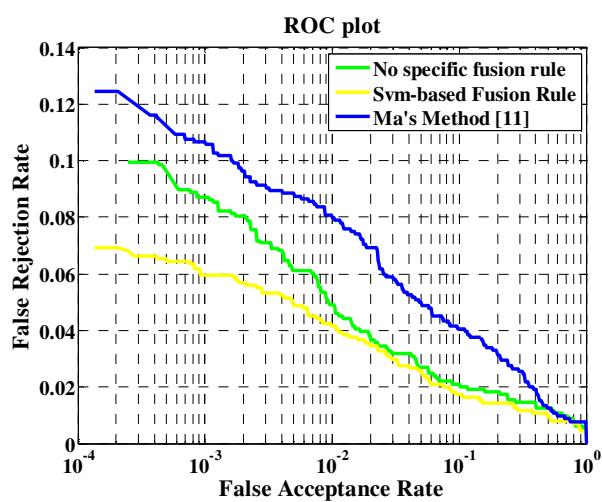

(b)

Fig. 2. This figure shows ROC plots of (a) first and (b) second session of UBIRIS. ROC curves are obtained from the proposed method without fusion rule, proposed method with SVM-based fusion, and Ma's method.

Table 1. Obtained results from both sessions of UBIRIS database

\begin{tabular}{|c|c|c|c|c|c|c|}
\hline \multirow{2}{*}{ Method } & \multicolumn{5}{|c|}{ Obtained results from } \\
\cline { 2 - 6 } & \multicolumn{3}{|c|}{ First session } & \multicolumn{2}{c|}{ Second session } \\
\cline { 2 - 6 } & EER) & $\begin{array}{c}\text { FRR, } \\
\text { FAR=0.001\% }\end{array}$ & EER (\%) & $\begin{array}{c}\text { FRR, } \\
\text { FAR=0.001\% }\end{array}$ \\
\hline $\begin{array}{c}\text { Poursaberi and } \\
\text { Araabi [19] }\end{array}$ & 2.1 & 7.6 & 5.0 & 16.3 \\
\hline Ma et al. [11] & 1.9 & 4.7 & 5.0 & 12.4 \\
\hline $\begin{array}{c}\text { Rakshit et al. } \\
\text { [20] }\end{array}$ & 1.2 & 5.5 & 3.8 & 8.8 \\
\hline $\begin{array}{c}\text { Ahmadi et al. } \\
\text { [21] }\end{array}$ & 1.9 & & 5.4 & & 8.0 & 21.6 \\
\hline $\begin{array}{c}\text { Proposed } \\
\text { method with } \\
\text { SVM-based } \\
\text { fusion rule }\end{array}$ & 0.4 & 1.8 & & 3.0 & 6.8 \\
\hline
\end{tabular}

on approximation coefficients of third level and removing the detailed coefficients of other frequency sub-bands. Based on our experiments, the useful information of denoised intensity signals were concentrated at the fifth dyadic scale of decomposition. Accordingly, we took the wavelet coefficients obtained through performing CWT on the specified scale and generate the corresponding binary code based on the signs of extracted coefficients. As a result, associated with an intensity signal, an iris code comprised of 256 bits was generated. Thus, a binary matrix consisting of five generated codes was created for a given iris image. 
To obtain fusion rules, five-fold cross validation was adopted. That is, database was divided to five subsets, and each time, one of the 5 subsets is used as the test set and the other 4 subsets are put together to form a subset for training of fusion rule. The final ROC is obtained from averaging five resulting ROCs. We followed abovementioned strategy to assess efficiency of proposed method in both sessions of the UBIRIS database.

Figure 2 shows ROCs of proposed method when (1) no specific fusion rule is adopted, (2) using a SVM-based fusion rule, and ROC of method suggested in [11]. This way of comparison enables us to highlight the role of fusion rule in our achievements. It should be noted that utilizing no specific fusion rule is the same as summing dissimilarities or to measure dissimilarity of iris patterns when all five generated codes simultaneously are incorporated in matching process and as a result in this case only one dissimilarity value is obtained when two iris patterns compared. As it is seen, while our contributions including de-noising strategy and defining overlapped patches make a significant prominence in comparison with Ma's method in both sessions, enhancement achieved by utilizing fusion rule is more outstanding in the second session of UBIRIS. This is due to less noise content of images contained in the first session of database and substantial uniform distribution of information over European iris textures captured in visible light illumination. Accordingly, combination of texture information coming from different areas of iris cannot provide a significant enhancement in the first session of UBIRIS.

To make a more comprehensive comparison, we also implemented the methods suggested in [19, 20, and 21] based on their publications and tuned their pertaining parameters to get best possible results. To have a quantitative comparison between our method and other implemented methods, EER and FRR (@ Far=.001\%) were computed and tabulated in Table 1 . As it is seen, proposed method not only gives a superior performance in comparison with local intensity variation method [11] but also achieves highest efficiency compared with other implemented methods. This prominence is more highlighted when one compares obtained results from second session of UBIRIS. This implies the effectiveness of our method facing highly degraded images especially those suffering from reflections of cornea.

\subsection{Robustness Evaluation}

In the previous subsection, we demonstrated efficiency of proposed method. In the following, our aim is to assess robustness of proposed approach dealing with degradation factors like Lack of focus owing to a large stand-off distance, blurring as a result of relaxing constraints imposed on the motion of the subject, deformation of texture caused by pupil expansion/ contraction.

Due to the subject of security, being invariant to such degradations is of critical importance. To analyze how our proposed method deals with these unconstraint conditions, we gathered a small but noisy iris database. It is comprised of 200 iris images from 20 subjects that severely suffer from deformation of texture. In order to approach non-cooperative recognition -as an extreme case of noisy recognition, lack of focus and subjects' movement are simulated in our experiments. We also model defects in CCD camera and degradations during transmission of images, by injecting Salt and Pepper noise into captured images. 
In each experiment, only one degradation factor is added to the iris images. In the case of blurring, one iris image of quality form per subject is selected and blurred with filters of 10 and 20 pixels length in four different directions $\left(0^{\circ}, 45^{\circ}, 90^{\circ}\right.$, and $\left.135^{\circ}\right)$. In the next experiment, Salt and Pepper noise with two noise densities $(0.05$ and 0.1 ) are injected to all captured images. We also model lack of focus in all iris images using circular averaging filters with point spread functions in the form of disks with 10 and 15 pixels radii. Verification results based on our experiments are shown in Table 2. As it is seen, except for motion blur of 10-pixel length, the proposed method exhibits the least performance degradation. Although our method has relatively small privilege over [11] in slight noisy condition, there exists a significant difference between achieved results and that of [11] in more noisy conditions. Furthermore, high robustness of feature extraction method versus the lack of focus and motion blur establishes our approach as an effective one to face highly degraded images that captured in non-ideal conditions.

Table 2. Obtained results through robustness evaluation of proposed method

\begin{tabular}{|c|c|c|c|c|c|c|c|c|c|c|c|c|}
\hline \multirow{5}{*}{ Method } & \multicolumn{12}{|c|}{ Degradation (\%) } \\
\hline & \multicolumn{8}{|c|}{ Motion Blur } & \multirow{3}{*}{\multicolumn{2}{|c|}{$\begin{array}{c}\text { Defocus } \\
\begin{array}{c}\text { Radius of } \\
\text { Disk }\end{array}\end{array}$}} & \multirow{3}{*}{\multicolumn{2}{|c|}{$\begin{array}{c}\begin{array}{c}\text { Noise } \\
\text { (Salt \& Pepper) }\end{array} \\
\text { Density }\end{array}$}} \\
\hline & \multirow{2}{*}{\multicolumn{4}{|c|}{$\begin{array}{l}\text { Length of the blur } \\
10 \text { pix } \\
\text { Degree of the blur }\end{array}$}} & \multirow{2}{*}{\multicolumn{4}{|c|}{$\begin{array}{l}\text { Length of the blur } \\
20 \text { pix } \\
\text { Degree of the blur }\end{array}$}} & & & & \\
\hline & & & & & & & & & & & & \\
\hline & 0 & 45 & 90 & 135 & 0 & 45 & 90 & 135 & 10 & 15 & $5 \%$ & $10 \%$ \\
\hline Ma et al. [11] & 0.5 & 1.7 & 0.7 & 1.7 & $\overline{6.6}$ & 12.7 & 8.7 & 11.8 & 3.6 & 8.2 & $1.9 \pm 0.8$ & $3.8 \pm 1.0$ \\
\hline Proposed & 1.2 & 1.0 & 1.3 & 2.0 & 3.4 & 6.4 & 4.5 & 5.1 & 1.0 & 3.7 & $1.2 \pm 0.4$ & $2.3 \pm 1.0$ \\
\hline
\end{tabular}

\section{Conclusion}

One of the main issues in iris recognition is coping with low quality iris images captured in uncontrolled lighting conditions and without active cooperative subject's behavior. In this paper, a modified version of local intensity variation method capable of facing mentioned challenges was proposed. Experimental results on the UBIRIS demonstrated the efficiency of proposed method in comparison with state-of-the-art algorithms. Our algorithm also provided more robustness in comparison with local intensity variation method.

\section{References}

[1] Proença, H., Alexandre, L.A.: UBIRIS: A noisy iris image database. In: Roli, F., Vitulano, S. (eds.) ICIAP 2005. LNCS, vol. 3617, pp. 970-977. Springer, Heidelberg (2005), http://iris.di.ubi.pt

[2] Krichen, E., Chenafa, M., Garcia-Salicetti, S., Dorizzi, B.: Color-Based Iris Verification. In: Lee, S.-W., Li, S.Z. (eds.) ICB 2007. LNCS, vol. 4642, pp. 997-1005. Springer, Heidelberg (2007) 
[3] Hosseini, S.M., Araabi, B.N., Soltanian-Zadeh, H.: Shape Analysis of Stroma for Iris Recognition. In: Lee, S.-W., Li, S.Z. (eds.) ICB 2007. LNCS, vol. 4642, pp. 790-799. Springer, Heidelberg (2007)

[4] Proença, H., Alexandre, L.A.: Iris Recognition: An Entropy-Based Coding Strategy Robust to Noisy Imaging Environments. In: Bebis, G., Boyle, R., Parvin, B., Koracin, D., Paragios, N., Tanveer, S.-M., Ju, T., Liu, Z., Coquillart, S., Cruz-Neira, C., Müller, T., Malzbender, T. (eds.) ISVC 2007, Part I. LNCS, vol. 4841, pp. 621-632. Springer, Heidelberg (2007)

[5] Lee, J., Huang, P.S., Chang, C., Tu, T.: A Novel Approach for Iris Recognition Using Local Edge Patterns. In: Bebis, G., Boyle, R., Parvin, B., Koracin, D., Paragios, N., Tanveer, S.-M., Ju, T., Liu, Z., Coquillart, S., Cruz-Neira, C., Müller, T., Malzbender, T. (eds.) ISVC 2007, Part II. LNCS, vol. 4842, pp. 479-488. Springer, Heidelberg (2007)

[6] Proença, H., Alexandre, L.A.: Toward Noncooperative Iris Recognition: A Classification Approach Using Multiple Signatures. IEEE Transaction on Pattern Analysis and Machine Intelligence 29(4), 607-612 (2007)

[7] Bashir, F., Casaverde, P., Usher, D., Friedman, M.: Eagle-Eyes ${ }^{T M}$ : A System for Iris Recognition at a Distance. In: Proceeding of IEEE Conference on Technologies for Homeland Security, pp. 426-431 (2008)

[8] Tajbakhsh, N., Araabi, B.N., Soltanian-zadeh, H.: An Intelligent Decision Combiner Applied to Noncooperative Iris Recognition. In: IEEE Proceedings of the 11th International Conference on Information Fusion, 6 pages (2008)

[9] Tajbakhsh, N., Araabi, B.N., Soltanian-zadeh, H.: Feature Fusion as a Practical Solution toward Noncooperative Iris Recognition. In: IEEE Proceedings of the 11th International Conference on Information Fusion, 7 pages (2008)

[10] Ma, L., Tan, T., Wang, Y., Zhang, D.: Efficient Iris Recognition by Characterizing Key Local Variations. IEEE Transactions on Image Processing 13(6), 739-750 (2004)

[11] Ma, L., Tan, T., Wang, Y., Zhang, D.: Local intensity variation analysis for iris recognition. Pattern recognition 37(6), 1287-1298 (2004)

[12] Proença, H.: Towards Non-Cooperative Biometric Iris Recognition. University of Beira Interior, Phd Thesis (January 2007) ISBN: 978-972-99648-2-4

[13] Poursaberi, A., Araabi, B.N.: A Half-Eye Wavelet Based Method for Iris Recognition. In: ISDA 2005, Proceeding of 5th International Conference on Intelligent Systems Design and Applications, pp. 262-267 (2005)

[14] Brunelli, R., Alavigna, D.: Person Identification Using Multiple Cues. IEEE Transactions on Pattern Analysis and Machine Intelligence 17(10), 955-966 (1995)

[15] Fierrez-Aguilar, J., Ortega-Garcia, J., Gonzalez-Rodriguez, J., Bigun, J.: Discriminative Multimodal Biometric Authentication based on Quality Measures. Pattern Recognition 38(5), 777-779 (2005)

[16] Nandakumar, K., Chen, Y., Dass, S.C., Jain, A.K.: Likelihood Ratio-Based Biometric Score Fusion. IEEE Transactions on Pattern Analysis and Machine Intelligence 30(2), 342-347 (2008)

[17] Prabhakar, S., Jain, A.K.: Decision-level Fusion in Fingerprint Verification. Pattern Recognition 35(4), 861-874 (2002)

[18] Griffin, P.: Optimal Biometric Fusion for Identity Verification. Identix Research, Technical Report RDNJ-03-0064 (2004) 
[19] Poursaberi, A., Araabi, B.N.: Iris Recognition for Partially Occluded Images: Methodology and Sensitivity Analysis. EURASIP Journal on Advances in Signal Processing 2007(1), 12 pages (2007)

[20] Rakshit, S., Monro, D.M., Zhang, D.: DCT-Based Iris Recognition. IEEE Transactions on Pattern Analysis and Machine Intelligence 29(4), 586-595 (2007)

[21] Ahmadi, H., Pousaberi, A., Azizzadeh, A., Kamarei, M.: An Efficient Iris Coding Based on Gauss-Laguerre Wavelets. In: Lee, S.-W., Li, S.Z. (eds.) ICB 2007. LNCS, vol. 4642, pp. 917-926. Springer, Heidelberg (2007) 